\title{
Ablation of recurrent primary liver cancer using ${ }^{131}$ I-lipiodol
}

\author{
Richard Novell, Andrew Hilson and Kenneth Hobbs
}

\begin{abstract}
Academic Department of Surgery and Department of Medical Physics, Royal Free Hospital School of Medicine, Pond Street, London NW3 2QG, UK
\end{abstract}

\begin{abstract}
Summary: A case of recurrent hepatocellular carcinoma following resection is reported. The patient received 2 treatments of ${ }^{131}$ I-lipiodol administered via the hepatic artery at angiography. Good localization in tumour tissue was observed on subsequent computed tomographic scans and no adverse effects were seen after either treatment. After the first treatment 2 nodules of recurrent tumour were resected and proved to be totally necrotic. The patient remains well 9 months later with a persistently elevated serum alphafetoprotein but no evidence of residual tumour. It is suggested that ${ }^{131}$ I-lipiodol may be the treatment of choice for small tumours in elderly high-risk patients.
\end{abstract}

\section{Introduction}

Although comparatively rare in Europe, hepatocellular carcinoma (HCC) is estimated to account for one million deaths worldwide each year. ${ }^{1}$ There is evidence that the occurrence is increasing in both western Europe and in the USA. At present only surgical resection or transplantation offer the prospect of long-term survival. Careful patient selection is crucial in resection, as survival in cirrhotic patients is significantly worse, ${ }^{2}$ and as a result the resectability rate in $\mathrm{HCC}$ may be as low as $1 \%{ }^{3}$ Over $75 \%$ of patients transplanted for $\mathrm{HCC}$ will be dead within 3 years, the vast majority from tumour recurrence. $^{4}$

For those patients with an irresectable tumour the prognosis is bleak, survival for more than 6 months being exceptional. ${ }^{5}$ A variety of nonsurgical treatments has been claimed to be of palliative benefit, including devascularization techniques, chemotherapy (used individually or in combination), cryotherapy and alcohol injection. There is, however, little evidence that any significantly improve survival.

We report a case of hepatocellular carcinoma treated with ${ }^{131}$ I-lipiodol, a new tumour-selective agent, in which histological evidence of complete tumour ablation was obtained.

\section{Case history}

A 65 year old Egyptian woman was found on routine screening to have an abnormally raised

Correspondence: J.R. Novell, M.A., F.R.C.S.

Accepted: 15 October 1990 serum alkaline phosphatase. The patient herself was asymptomatic but clinical examination demonstrated a palpable hepatic margin. Computed tomographic (CT) scanning revealed a solitary mass in the right hepatic lobe. The serum alpha fetoprotein (AFP) was markedly raised at $335 \mathrm{kU} / \mathrm{l}$. A biopsy of the surrounding liver showed fatty change but no evidence of cirrhosis. A chest X-ray and bone scan were unremarkable.

A clinical diagnosis of hepatocellular carcinoma was made, and, in view of the patient's good general health and liver function, a local resection of the tumour was performed, incorporating a $1 \mathrm{~cm}$ margin of uninvolved liver tissue. The patient made an excellent recovery and left hospital 1 week later. Histological examination of the resected specimen confirmed the diagnosis of hepatocellular carcinoma. Postoperatively the AFP fell to within normal limits.

The patient remained well for 3 years, when her AFP once again became elevated at $92 \mathrm{kU} / \mathrm{l}$. A lipiodol-enhanced CT scan demonstrated a further lesion in the tip of the left hepatic lobe. Four hundred and seventy-five $\mathrm{mBq}$ of ${ }^{131}$ I-lipiodol (CIS[UK]) was administered via the hepatic artery at angiography; a CT scan performed 10 days later showed good localization of the lipiodol within the tumour (Figure 1). Gamma scintigraphy performed at 2 and 9 days confirmed retention of activity at the site of the tumour. The volumes of the tumour nodules were calculated from the CT scan using the formula

Volume $=$ maximum tumour diameter $x$ perpendicular diameter ${ }^{2}$ 


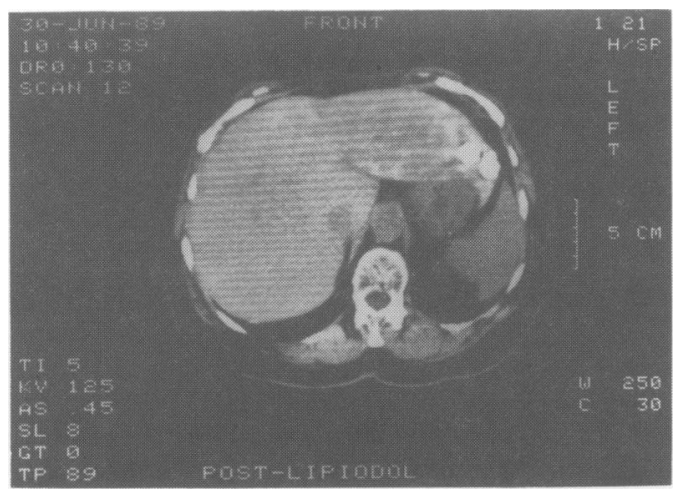

Figure 1 CT scan of liver showing ${ }^{131}$ I-lipiodol uptake by tumour nodules.

and found to be $4.3 \mathrm{~cm}^{3}$ and $9.3 \mathrm{~cm}^{3}$ respectively. Using distribution data from previously published studies, ${ }^{6,7}$ the dose of radioactivity administered to the tumour was calculated to be approximately $100 \mathrm{~Gy}$. The patient suffered no side effects from the treatment and her haematological indices remained within normal limits.

She remained asymptomatic and a repeat CT scan 3 months later showed no increase in the size of the tumour. The serum AFP, however, remained raised at $160 \mathrm{kU} / 1$. In view of these findings a second local resection was performed and a $5 \mathrm{~cm}$ wedge of liver containing 2 discrete tumour nodules removed. The actual volumes of the nodules following ${ }^{131}$ I treatment were $1.7 \mathrm{~cm}^{3}$ and $4.0 \mathrm{~cm}^{3}$ respectively. Use of the intra-operative ultrasound probe demonstrated a further mass lying posteriorly between the right and middle hepatic veins, which was unresectable. Aspiration cytology was performed under ultrasound control.

The postoperative course was once again uneventful. Histology on the resected specimen showed both tumour nodules to be completely necrotic and surrounded by a zone of fibrotic but non-malignant parenchyma (Figure 2). Cytological examination of the aspirate obtained at laparotomy confirmed the posterior mass to be a further deposit of hepatocellular carcinoma. Two weeks later the patient received a further $632 \mathrm{mBq}$ of intra-arterial ${ }^{131}$ I-lipiodol, again without any evidence of toxicity. Subsequent gamma scans again showed retained activity in the region of the tumour.

The patient remains asymptomatic 9 months later with no evidence of extrahepatic metastases. A follow-up CT scan at 3 months showed no evidence of residual tumour posteriorly, but demonstrated a further lesion adjacent to the right branch of the portal vein (Figure 3a). On repeat scanning 6 months later the lesion is unchanged (Figure 3b); ultrasound scanning has shown regen-

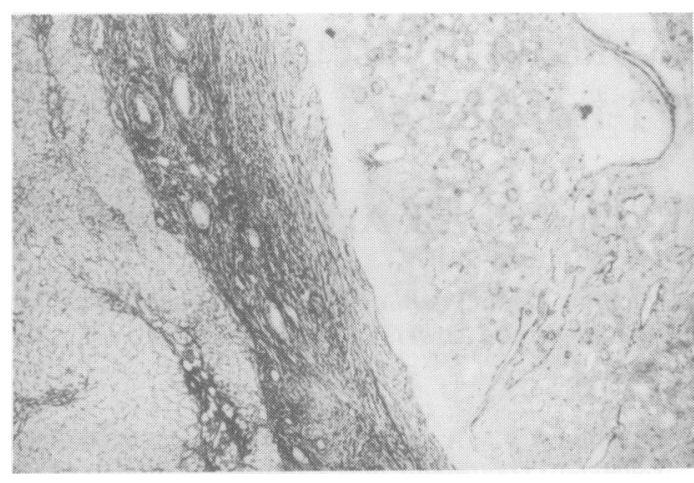

Figure 2 Section of resected nodule showing necrotic tumour surrounded by zone of fibrotic non-neoplastic liver cells (Reticulin stain). Left: normal liver architecture; middle: zone of fibrosis and chronic inflammatory infiltrate; right: necrotic tumour nodule.

a

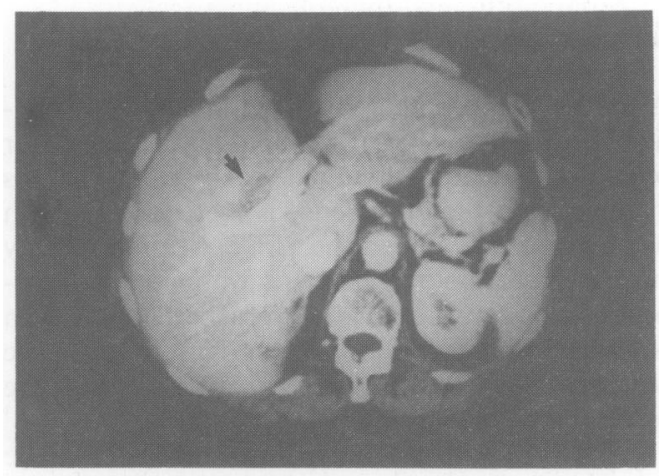

b

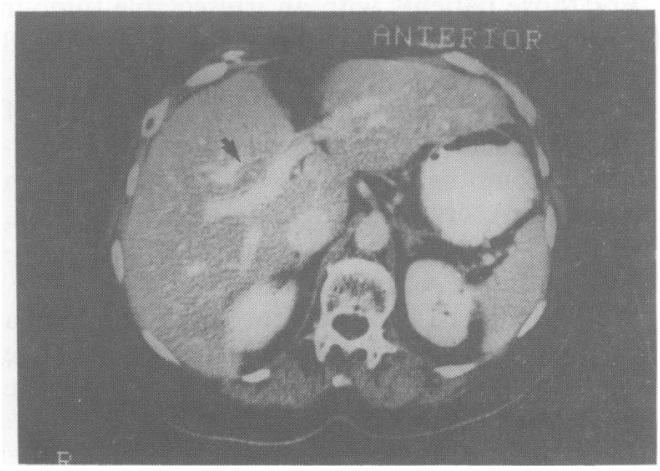

Figure 3 Lesion adjacent to right branch of portal vein: (a) 3 months after ${ }^{131}$ I-lipiodol treatment; (b) 6 months later.

erative nodules throughout the lobe but no evidence of focal tumour. The serum AFP has remained elevated at $200-300 \mathrm{kU} / 1$ throughout the follow-up period without a consistently upward 
trend. On the basis of these data the most likely cause for the CT abnormality is a regenerative nodule: no further ${ }^{131}$ I-lipiodol treatments are planned.

\section{Discussion}

Lipiodol Ultra Fluid (May \& Baker, UK) is a stable iodine-containing lipid derived from poppyseed oil, which has been used for many years as a lymphographic contrast medium. When injected into the hepatic artery it is selectively retained by hepatocellular carcinomas, and is particularly effective in demonstrating small nodules of tumour. ${ }^{8}$ This property of selective retention makes it an ideal vehicle for tumour targeting, and Lipiodoltargeted chemotherapy is currently the treatment of choice for unresectable hepatocellular carcinoma in many Japanese centres. ${ }^{9,10}$ More recently it has been used as a means of targeting radiotherapy to hepatic tumours, by replacing a fraction of the iodine component by the isotope ${ }^{131}$ I. Scintigraphy in human subjects has shown a similar preferential uptake and retention to the non-radioactive lipid. ${ }^{11}$ Reports from clinical trials have been encouraging, with symptomatic improvement in the majority of patients and reduction in tumour size and ascites. ${ }^{12}$

\section{References}

1. London, W. Primary hepatocellular carcinoma - etiology, pathogenesis and prevention. Hum Pathol 1981, 12: 10851097.

2. Nagasue, N., Yukaya, H., Kohno, H., Chang, Y. \& Nakamura, T. Morbidity and mortality after major hepatic resection in cirrhotic patients with hepatocellular carcinoma. HPB Surg 1988, 1: 45-56.

3. Maraj, R., Kew, M.C. \& Hyslop, R.J. Resectability rate of hepatocellular carcinoma in rural southern Africans. $B r J$ Surg 1988, 75: 335-338.

4. Iwatsuki, S. \& Starzl, T.E. Liver transplantation in the treatment of liver cancer. In: Okuda, K. \& Ishak, K.G. (eds) Neoplasms of the Liver. Springer-Verlag, Tokyo, 1987, pp. 397-405.

5. Takayasu, K., Shima, Y., Muramatsu, Y. et al. Hepatocellular carcinoma: treatment with intra-arterial iodized oil with and without chemotherapeutic agents. Radiology 1987, 162: 345-351.

6. Madsen, M., Park, C. \& Thakur, M. Dosimetry of Iodine-131 ethiodol in the treatment of hepatoma. J Nucl Med 1988, 29: $1038-1044$.

7. Nakajo, M., Kobayashi, H., Shimabukuro, K. et al. Biodistribution and in vivo kinetics of iodine-131 lipiodol infused via the hepatic artery of patients with hepatic cancer. J Nucl Med 1988, 29: 1066-1077.

8. Hayashi, N., Yamamoto, K., Tamaki, N. et al. Metastatic nodules of hepatocellular carcinoma: detection with angiography, CT, and US. Radiology 1987, 165: 61-63.
Our own experience in 8 patients to date has confirmed these findings. ${ }^{13}$

To date there have been no reports of total tumour ablation using radioactive lipiodol. In this case, while the original malignant foci were rendered completely necrotic after treatment, the persistence of a raised serum AFP has raised the possibility of residual tumour. However, the patient remains asymptomatic and neither repeated CT scans, with and without Lipiodol enhancement, nor ultrasound scanning have demonstrated a further malignant focus. Persistent elevation in serum AFP has been documented after curative hepatic resection, and may be related to regenerative nodules in the cirrhotic liver. ${ }^{14}$

This case demonstrates that ${ }^{131}$ I-lipiodol is sapable of delivering an ablation dose of radiation to small foci of hepatocellular carcinoma. Lipiodol-targeted radiotherapy may be the treatment of choice for small tumours in older, cirrhotic patients who are at high risk of complications following surgical resection.

\section{Acknowledgement}

The authors wish to thank CIS Bioindustries for supplying the ${ }^{131}$ I-lipiodol used in this case.

9. Kanematsu, T., Furuta, T., Takenaka, K. et al. A 5 year experience of lipiodolization: selective regional chemotherapy for 200 patients with hepatocellular carcinoma. Hepatology 1989, 10: 98-102.

10. Ohishi, H., Uchida, H., Yoshimura, H. et al. Hepatocellular carcinoma detected by iodized oil; use of anticancer agents. Radiology 1985, 154: 25-29.

11. Raoul, J., Bourguet, P., Bretagne, J. et al. Hepatic artery injection of I-131 labelled lipiodol. Part I: biodistribution study results in patients with hepatocellular carcinoma and liver metastases. Radiology 1988, 168: 541-545.

12. Bretagne, J., Raoul, J., Bourguet, P. et al. Hepatic artery injection of 131-I labelled Lipiodol. Part II: preliminary results of therapeutic use in patients with hepatocellular carcinoma and liver metastases. Radiology 1988, 168: 547-550.

13. Novell, J.R., Mistry, P., Reddy, K., Hilson, A. \& Hobbs, K.E.F. Targeted treatment of primary liver cell carcinoma. HPB Surg 1990, 2 (Suppl): 85.

14. Ezaki, T., Yukuya, H., Ogawa, Y., Chang, Y.\& Nagasue, N. Elevation of alpha fetoprotein level without evidence of recurrence after hepatectomy for hepatocellular carcinoma. Cancer 1988, 61: 1880-1883. 